\title{
A Real-time Algorithm Based on Triaxial Accelerometer for the Detection of
}

\author{
$\mathrm{Na} \mathrm{Li}$ \\ Embedded software and systems \\ institute \\ Beijing University of technology \\ Beijing China \\ nana439@emails.bjut.edu.cn
}

\section{Human Activity State}

\author{
Yibin Hou \\ Embedded software and systems \\ institute \\ Beijing University of technology \\ Beijing China \\ ybhou@bjut.edu.cn
}

\author{
Zhangqin Huang \\ Embedded software and systems \\ institute \\ Beijing University of technology \\ Beijing China \\ zhuang@bjut.edu.cn
}

\begin{abstract}
Assessment and identification of human activity and posture with triaxial accelerometer can provide information about the health state and the chronic care. This paper proposes a human state recognition algorithm based on Kalman filter (SRKF), which could identify steady state and state transition in real time. In this study, an automatic state-recognition system consisting of a Bluetooth module and a smart phone is developed. The Bluetooth module with a triaxial accelerometer was placed on the body and the raw sensor data was transported to and processed on the smart phone. Associated kinematics characteristic of human activity and the accelerometer signal, the results of amplitude function of vector CSVM were processed by Kalman filter to identify human state. Data were collected from ten adults in unsupervised environment. Experiment result shows that the algorithm has achieved better performance on the smart phone with limited computing and storage capacity.
\end{abstract}

\section{Keywords}

triaxial accelerometer, CSVM, Kalman filter.

\section{INTRODUCTION}

Technical progress in microelectronics provides a new lowweight, low-power consumption miniaturized sensing device, which also allows integrating sensors, micro control unit and wireless transmission modules into a single unit to be worn by people everyday living. This system can further open the door to a world of healthcare applications, such as fitness monitoring, eldercare support, long-term preventive and cognitive assistance physiological impairments, and is essential to enabling contextaware computing in pervasive computing scenarios [1]. Accelerometers have been shown to be especially suitable for estimating the human activity [2]. Previous studied have shown that it is possible to infer the daily activity with good accuracy using some methods based on accelerometer measurements.

Most studies have been carried out on the features of accelerometer signal and recognition techniques. As for abstracting features, researches have been carried out based on the physical characteristics of signal and kinematics characteristic of

Permission to make digital or hard copies of all or part of this work for personal or classroom use is granted without fee provided that copies are not made or distributed for profit or commercial advantage and that copies bear this notice and the full citation on the first page. To copy otherwise, to republish, to post on servers or to redistribute to lists, requires prior specific permission and/or a fee.

BODYNETS 2011, November 07-08, Beijing, People's Republic of China

Copyright (c) 2012 ICST 978-1-936968-29-9

DOI 10.4108/icst.bodynets.2011.247166 human. In terms of the physical characteristics of signal, timedomain parameters and frequency-domain parameters are extracted. Time-domain parameters include mean value, variance, standard deviation [3] and so on. Frequency-domain parameters include Fourier transform coefficients [4], spectrum entropy [5] and so on. About kinematics characteristic of human, parameters such as: signal vector magnitude [6], title angle [7] are extracted. As for the recognition techniques, many classification methods have been used, such as: Bayesian networks [8], neural networks [8], support vector machine [9], Gaussian mixture models [10]; the threshold classification, Fisher's linear discriminant [11].

In actual practice, these methods are always mixed together. E.g.: Adil Mehmood Khan [12] has used AR model to represent the relationship of signals, then abstracted augmented-feature vector that consists of seven property value, in addition, lineardiscriminant analysis was used to classify states transition and artificial-neural nets was applied to identify specific activity. David Curone [13] has abstracted feature vector, e.g.: signal magnitude area (SMA), title angle (TA), combining with several rules to obtain the classification. The main drawback of above methods is that they need abstracting multiple features and rules to process amount of data, which is limited on the terminal mobile device.

In previous studies, based on their complexity, activities are categorized into two levels: gesture and action. Gestures are elementary movements of a person's body part. E.g.: "stretching an arm", "raising a leg". Actions are single-person activities that may be composed of multiple gestures organized temporally, such as "walking", "waving", and "punching" [14]. The paper focuses on "action", and here it is named "activity". We pay attention to the state. State is a general situation that the whole body is being for a relatively long period of time, which may be composed of several activities, e.g.: state of motion, static and so on. States of human are categorized into two classes: steady state and nonsteady state. Continuous activities are regarded as steady states, for example: standing, running, walking and so on; if there is a transition between different activities, then it is a non-steady state, for example: running-to-walking, standing-to-walking. The objective of this paper is to identify the key state. Experiments indicate when the gestures are repetitively, the tendency of changes in acceleration is periodic. So we ignore the specific gesture that cause changes of acceleration at some point, and take account of the changes of acceleration caused repetitive activities. In the paper, the algorithm that detecting human state based Kalman filter (SRKF) is proposed. It uses the changes of signal magnitude vector (CSVM) as quantity of state, then applies Kalman filter to process characteristic values in order to recognize the steady and non-steady state in real-time, which further contributes to identify specific activity. The algorithm has run on 
the smart phone with Android platform and has proved the validation.

The rest of paper is organized as follows. Section 2 presents design of the algorithm SRKF. Section 3 presents the system of experiment and the experimental evaluation. Section 4 concludes the paper with a summary and the future work.

\section{METHOD}

\subsection{Feature Extract}

In order to simplify complex of the vector operation, we use the signal vector magnitude (SVM), which reflects intensity of changes in accelerations [6].

$$
S V M_{t}=\sqrt{a_{x, t}^{2}+a_{y, t}^{2}+a_{z, t}^{2}}
$$

Based on the characteristic of human's actions, we extract the extreme point of SVM, which can be obtained when continuing to read raw sensor output and detect the peak of the SVM. In order to reduce the computing overhead, we use the change of SVM (CSVM) as quantity of state, which reflects the trends of absolute value of SVM. When a new signal arrives, it will be compared with the former one, if the result is greater than zero, which indicates CSVM is still increasing, then we ignore this sample and wait for the next signal and then repeat the comparison; but if the result is less than zero, which indicates the signal is peak, and we marks it. By this way, we can obtain the value of CSVM by calculating the difference between the peaks. We regard CSVM as the feature quantification.

$$
C S V M_{t}=\operatorname{Max}\left(S V M_{t}\right)-\operatorname{Min}\left(S V M_{t-k}\right)
$$

Because human activities are complex and sensor has high sensitivity, so the collected signals have varying amounts of noise The tiny movements or friction of body can cause the minimal changes in SVM, and will result much CSVM with small absolute value, which do not represent the real activities of body. So these points should be ignored, that means, if the CSVM satisfies equation (3), then this CSVM is deserted and is not used as quantity of state, otherwise, the points are collected.

$$
\operatorname{CSVM}_{t+k}<0.2 g
$$

When using the equation 3, we find when the human body stays static, the value of CSVM is very small and is close to zero. But they reflect current state, which should not be ignored. That is, if the absolute value of CSVM is less than $0.2 \mathrm{~g}$ and happens consecutively $\mathrm{K}$ times, then we take these samples as useful. In experiment, the value of $\mathrm{K}$ associates with sampling rate. In fact, if the samples satisfy the equation 3 and happen consecutively for more than 0.5 seconds, we regard the samples as effective, which indicates the body is static.

\subsection{Identification Algorithm}

The paper uses CSVM as quantity of state, which is modeled as an AR (1) process (an autoregressive model).

$$
X_{t}=\rho_{t-1, t} X_{t-1}+\zeta_{t-1}
$$

$\mathrm{Xt}$ : the quantity of state at $\mathrm{t}$ time; $\rho_{t-1, t}:$ state transition matrix from the time t-1 to $\mathrm{t} ; \zeta_{t-1}$ : the discreet white noise with zero mean and variance $\mathrm{Q}$.
According to the above analysis, sum of N CSVM is regarded as the $\mathrm{Xt}$, that is:

$$
X_{t}=F\left(\operatorname{CSVM}_{k}\right)=\sum_{k=1}^{n} \operatorname{CSVM}_{k} \quad \mathrm{k}=1,2 \ldots \mathrm{n}
$$

In this paper, we use least square method to estimate the parameter $\rho_{t-1, t}$ and Q, as follows:

$$
\begin{aligned}
& \rho_{t-1, t}=\left(M^{T} M\right)^{-1} M^{T} Z \\
& Q_{t-1, t}=\frac{1}{N-1}\left[Z-M \rho_{t-1, t}\right]^{T}\left[Z-M \rho_{t-1, t}\right] \\
& M=\left[0, X_{1,} X_{2, \ldots} X_{t-1}\right]^{T}, Z=\left[X_{1} X_{2, \ldots} X_{t}\right]^{T}
\end{aligned}
$$

It is not necessary to calculate $\rho_{t-1, t}$ and $Q_{t-1, t}$ adaptively at every time. We find that $\rho_{t-1, t}$ and $Q_{t-1, t}$ remain constant when the model does not contain much noise. When the state of body comes to steady, according to equation 6 , value of $\rho_{t-1, t}$ is close to 1 . In order to calculate efficiently, we regard $\rho_{t-1, t}=1$

Value of CSVM reflects the trend of change of acceleration. The bigger of CSVM, it means the change of acceleration is much larger than before, which is a state transition. In order to fit the trends, we use Kalman filter [15] to dispose.

$$
\left\{\begin{array}{l}
X_{t}=\rho_{t-1, t} X_{t-1}+\zeta_{t-1} \\
Y_{t}=H_{t} X_{t}+V_{t}
\end{array}\right.
$$

$V_{t}$ : the measurement noise with zero mean and variance R; $H_{t}=I$;

From the equation 8 , we can get the following results:

$$
\begin{aligned}
& \hat{X}_{t}=X_{t}^{\prime}+K_{t}\left(Y_{t}-H X_{t}^{\prime}\right) \\
& P_{t}^{\prime}=\rho_{t-1, t} P_{t-1} \rho_{t-1, t}^{\prime}+Q_{t-1} \\
& P_{t}=\left(I-K_{t} H\right) P_{t}^{\prime} \\
& K_{t}=P_{t}^{\prime} H^{T}\left(H P_{t}^{\prime} H^{T}+R_{t}\right)^{-1}
\end{aligned}
$$

$\hat{X}_{t}$ : posteriori estimation at t time, $P_{t}$ :posteriori estimate error covariance; $X_{t}^{\prime}$ : prior estimation at $\mathrm{t}$ time, $P_{t}^{\prime}:$ prior estimate error covariance; $X_{t}$ : real value at t time; Kt: the gain.

The measurement noise in equation (8) has the characteristic of Gaussian white noise. In practice, we should get statistical characteristics of it. Because the measurement noise exists in every state, so we can get its mean and variance by analyzing the statistical characteristics of the samples. In this paper, it assumed that people move from a still state, so the initial state of system is the still state of human, this means that the mean and variance of initial acceleration is zero and $\mathrm{R}$ respectively. That is: $\hat{X}_{0}=0$, $P_{0}=R$

When body comes to the steady state, $\hat{X}_{t}$ comes to a constant. So we can identify the state according to the value of $\hat{X}_{t}$. According to the experiments and analysis, we can get: 
(1) $\hat{X}_{t}<0.2 \mathrm{~g}$, the body stays a static state ;

(2) $\left(\hat{X}_{t}-\hat{X}_{t-k}\right)<0.5 g, \forall k \in[0 \ldots . i]$, the body stays a steady state, that means human repeats an activity;

(3) $\left(\hat{X}_{t}-\hat{X}_{t-k}\right)>0.5 g, \forall k \in(0 \ldots i]$, the human stays a nonsteady state, that means there is a state transition;

In above equations, parameter $\mathrm{i}$ associates with the sampling rate. When the signal satisfies the equation and arrives continually in 0.5 seconds, we think it is available.

\section{EXPERIMENT AND RESULTS}

In this section, we conduct experiments to evaluate whether the algorithm is effective for detecting the state of human. We first describe the experiment setup, and then analyze the evaluation results.

\subsection{Experimental Setup}

The experimental system consists of a measure unit, a Bluetooth module and a processing unit. We choose ADXL345 to compose the measure unit, which is a small, thin, low power, 3axis accelerometer with high resolution measurement at up to \pm 16 g. Digital output data is formatted as 16-bit two complement and is accessible through either a SPI or I2C digital interface, here we choose the I2C interface. The sampling frequency was $20 \mathrm{HZ}$ and the range of the sensor output was $\pm 8 \mathrm{~g}$, which satisfies the needs of human's daily life. According to the previous study, we know that the acceleration is affected by the sensor's location and attachment. The upper body suffers less influence caused by the movement of hands or legs, so it can reflect the state of whole body. But sensor on the hip or waist is affected much by the movements of legs, which contains much information about the gait style. In the paper, we fix the accelerometer on the chest level and waist respectively and compare the results. The Bluetooth model is responsible for transmitting signal; the Bluetooth chip used here is CSR-57E687BU723AN, which performs high processing speed. There are two units in the chip, they are DSP and VM. In order to reduce the delay time on transmitting, we choose DSP to get the samples from the register of the accelerometer. The central device in data processing and activity recognition is a Smartphone (I858, Shanghai) with Android 2.2, its CPU is $621 \mathrm{MHZ}$ and memory is $200 \mathrm{MB}$.

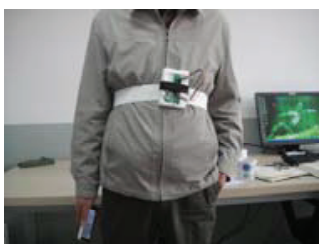

Figure 1. placement of the sensor

\subsection{Experimental Results}

Sessions of trials were realized to assess the performance of algorithm. Ten healthy adult subjects (five male and five female, $1.70 \mathrm{~m}$ average height, aged 27 years, no obvious disease on the leg) were asked to perform according to the activity plan (see table 1). The experiments were carried out in unsupervised environments, which can reflect human's daily life really. In the experiment environment, the ground is flat and there is no obvious slope, the stairs with 13 steps is 0.1 -meter height and the chair is 0.4-meter high with a soft cover. The subject's pace is for 100 to 110 strides in 60 seconds. The sample frequency is $20 \mathrm{HZ}$; we got 2000 data sets about one activity. The accelerometer were placed on top chest and waist respectively, the smart phone were taken on the subject's hands or placed on his pocket. When the subject performed the following activities: standing quiet$>$ walking with normal speed->sitting $->$ walking with normal speed->standing quiet, the results are as follows. Among these figures, from 2 to 4 are the results that the subject wearing the accelerometer on the chest; others were the results that the subject wearing the accelerometer on the waist.

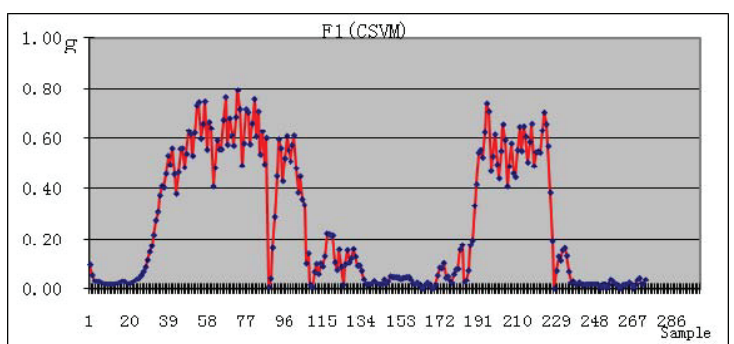

Figure 2 result of sum of 1 CSVM (on the chest)

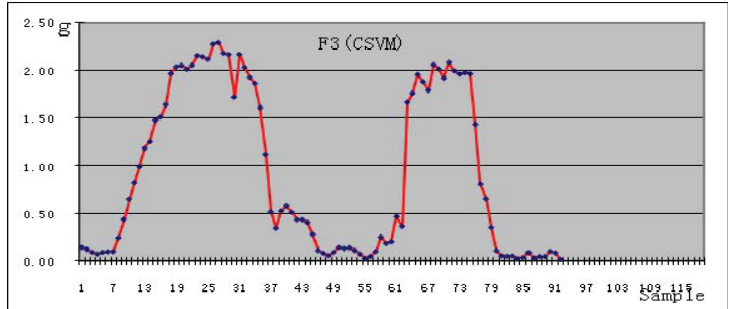

Figure 3. result of sum of 3 CSVM (on the chest)

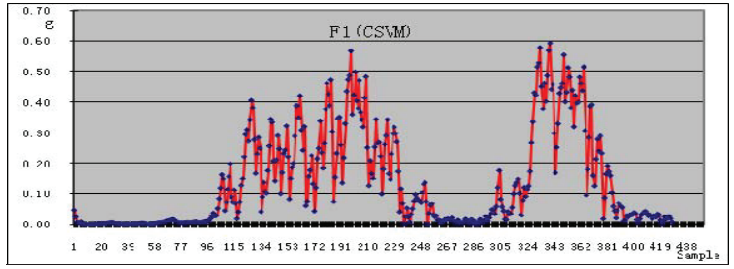

Figure 4. result of sum of 1 CSVM (on the waist)

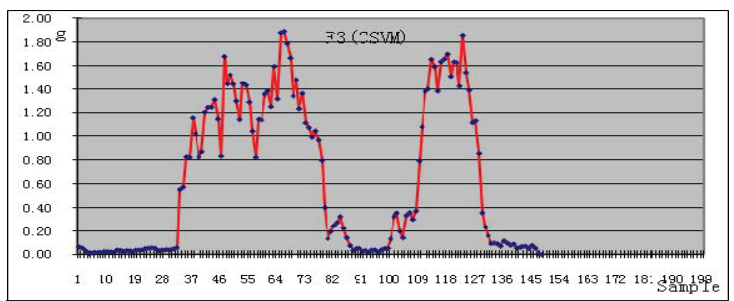

Figure 5. result of sum of 3 CSVM (on the waist)

From above figures, we know that placement location is one of important factors affecting the results. The accelerometer placed on chest reflects the whole body' activities more precise than that on the waist. On the figures, the rising or dropping of the curve indicates that the human's state is changing, smooth curve indicates that the human's state is steady, e.g. continuous walking; the curve almost overlapping with the horizontal axis indicates that human is static. Meanwhile, we find that processing result of sum of 3 CSVM is better than that of 1 CSVM. From analysis kinematics characteristic, we find that there are two moments when feet strike the ground that causes two wave peaks on the diagram of SVM on one gait cycle. So we use the sum of 3 CSVM as the quality of state. 
When the subject did activities: standing-> walking with a fast speed- >walking and with a slow speed-standing, the results performed as figure 8 :

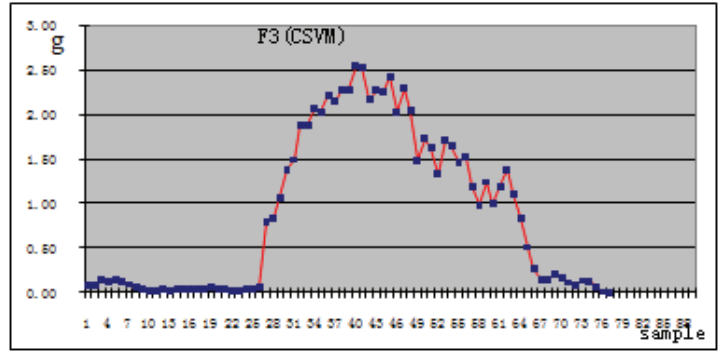

Figure 6. result of different rate(on the chset)

From the figure 8 , we can see that if the subject performs the same activity but with different rate, e.g. walking with a fast/slow rate, there is an obvious difference on the result processed by the algorithm. We find that the algorithm regards the same activity with different rate as the different one. The reason is that the value of CSVM is different, and the algorithm thinks there is a state transition.

Table 1. comparison of accuracy rate by using the SRKF algorithm and the threshold algorithm when placing sensor on the chest

\begin{tabular}{|c|c|c|}
\hline activity & $\begin{array}{c}\text { SRKF } \\
\text { algorithm }\end{array}$ & $\begin{array}{c}\text { SVM threshold } \\
\text { algorithm }\end{array}$ \\
\hline Walk-downstairs-walk & $95.00 \%$ & $90.00 \%$ \\
\hline Walk-upstairs-walk & $95.00 \%$ & $90.00 \%$ \\
\hline walk-run-stand & $94.00 \%$ & $91.3 \%$ \\
\hline stand-sit-stand & $97.30 \%$ & $95.00 \%$ \\
\hline $\begin{array}{c}\text { walk-downstairs- } \\
\text { walk-upstairs }\end{array}$ & $93.00 \%$ & $91.7 \%$ \\
\hline $\begin{array}{c}\text { Walk slow-walk fast- } \\
\text { run }\end{array}$ & $95.00 \%$ & $89.00 \%$ \\
\hline Stand quiet & $87.00 \%$ & $80.00 \%$ \\
\hline
\end{tabular}

From table 1, we can find that the SRKF algorithm has better accuracy in unsupervised environment than the threshold algorithm.

\section{CONCLUSION}

According to the kinematics characteristics of human, the paper proposes an algorithm to identify the state of human. Experimental results show that the method is better in accuracy and real-time than the traditional ways which only use threshold of acceleration. Now identification of human activities by using accelerometer is still at the experimental stage, the reason is that experiments are restricted and simple in the lab environment; (2) the algorithm was always processed on the small samples, that is, the number of subjects were always less than 50 and the continuous sampling time were less one month. The future work is to improve the hardware (suitable for integration devices in a jacket) and develop simple classification algorithm to identify the specific activity on a battery-powered mobile device.

\section{REFERENCES}

[1] Yu Huang,Jiannong Cao,Beihong Jin,et al. Flexible Cache Consistency Maintenance over Wireless Ad Hoc Networks,
IEEE Transactions on Parallel and Distributed Systems,2010,21(8),1150-1161

[2] M.Mathie, A. Coster,N. Lovell. Accelerometry: Providing an integrated, practical method for long-term, ambulatory monitoring of human movement, Physiol. Meas, vol. 25, 2004, 1-20,

[3] Stephen J Preece, John Y Goulermas. Activity identification using body-mounted sensors-a review of classification techniques, IOPScience, Physiol.Meas.30, 2009, R1-R33

[4] M.Ermes, J.Parka, J.Mantyjarvi, et al. Detecion of daily activities and sports with wearable sensors in controlled and uncontrolled conditions, IEEE Trans Inf. Technol. Biomed,vol.2, no.1, 2008, 20-26

[5] Ming Li, Gautam Thatte. Multimodal Physical Activity Recognition by Fusing Temporal and Cepstral Information, IEEE Transactions on neural systems and rehabilitation engineering, VOL.18,NO.4, 2010, 369-380

[6] D. M. Karantonis, M. R.Narayanan. Implementation of a real-time human movement classifier using a tri-axial accelerometer for ambulatory monitoring, IEEE Trans. Inf. Technol. Biomed., vol. 10, no. 1,2006, 156-167

[7] P. H. Veltink, H. B. J. Bussmann, W. de Vries, et al. Detection of static and dynamic activities using uniaxial accelerometers, IEEE Trans. Rehabil. Eng., vol. 4, no. 4, 1996, 375-385

[8] L. Bao, S. S. Intille. Activity recognition from userannotated acceleration data in Pervasive Computing, Berlin/Heidelberg, Germany: Springer-Verlag, 2004, 158175 .

[9] D. Giansanti, V. Macellari, G.Maccioni. New neural network classifierof fall-risk based on the Mahalanobis distance and kinematic parameters assessed by a wearable device, Physiol. Meas., vol. 29,2008, 11-19

[10] N. Ravi,N.Dandekar, P. Mysore, et al. Activity recognition from accelerometer data", in Proc. 20th Nat. Conf. Artif. Intell., 2005,1541-1546

[11] F. Allen, E. Ambikairajah, N. Lovell, et al. Classification of a known sequence of motions and postures from accelerometry data using adapted Gaussian mixture models, Physiol. Meas., vol. 27,2006, 935-951

[12] Adil Mehmood Khan, Young-Koo Lee. A Triaxial Accelerometer-Based Physical-Activity Recognition via Augmented-Signal Features and a Hierarchical Recognizer, IEEE trans on information technology in biomedicine,VOL.14, NO.5, 2010, 1166-1172

[13] Davide Curone, Gian Mario Bertolotti. A Real-Time and Self-Calibrating Algorithm Based on Triaxial Accelerometer Signals for the Detection of Human Posture and Activity, IEEE trans on information technology biomedicine, VOL.14, NO.4, 2010,1098-1105

[14] J.K.AGGARWAL, M.S.RYOO. Human Activity Analysis: A Review, ACM Computing Surveys, Vol.43, No.3, Article 16,2011

[15] R.E.Kalman. A new approach to linear filtering and prediction problems, J.Basic Eng, 1960, 35-45 